\title{
Prevalence of blindness in Western Australia: a population study using capture and recapture techniques
}

\author{
Julie Crewe, ${ }^{1}$ William H Morgan, ${ }^{2}$ Nigel Morlet, ${ }^{1}$ Antony Clark, ${ }^{1}$ Geoffrey Lam, ${ }^{3}$ \\ Richard Parsons, ${ }^{4}$ Aqif Mukhtar, ${ }^{1}$ Jonathon Ng, ${ }^{1}$ Margaret Crowley, \\ James Semmens ${ }^{1}$
}

\begin{abstract}
- Additional materials
(supplementary tables 2, 3 and 4 and supplementary figures 3 and 4) are published online only. To view these files please visit the journal online (http://bjo. bmi.com).

${ }^{1}$ Centre for Population Health Research, Curtin Health Innovation Research Institute, Curtin University, Perth, Australia

${ }^{2}$ Lions Eye Institute, Centre for Ophthalmic and Vision Science, University of Western Australia, Perth, Australia

${ }^{3}$ School of Paediatrics and Child Health, University of Western Australia, Perth, Australia ${ }^{4}$ School of Occupational Therapy and Social Work, Health Sciences, Curtin University, Perth, Australia

${ }^{5}$ The Association for the Blind of Western Australia, Perth, Australia
\end{abstract}

\section{Correspondence to Julie Crewe, Centre for Population Health Research, Curtin Health Innovation Research Institute, Curtin University, GP0 Box U1987. Perth, Western Australia 6845; j.crewe@curtin.edu.au}

Accepted 23 October 2011

\section{ABSTRACT}

Aim To determine the prevalence of blinding eye disease in Western Australia using a capture and recapture methodology.

Methods Three independent lists of residents of Western Australia who were also legally blind were collated during the capture periods in 2008-9. The first list was obtained from the state-wide blind register. A second list comprised patients routinely attending hospital outpatient eye clinics over a 6 -month period in 2008. The third list was patients attending ophthalmologists' routine clinical appointments over a 6-week period in 2009. Lists were compared to identify those individuals who were captured on each list and those who were recaptured by subsequent lists. Log-linear models were used to calculate the best fit and estimate the prevalence of blindness in the Western Australian population and extrapolated to a national prevalence of blindness in Australia.

Results 1771 legally blind people were identified on three separate lists. The best estimate of the prevalence of blindness in Western Australia was $3384195 \% \mathrm{Cl}$ 2947 to 3983 ) or $0.15 \%$ of the population of 2.25 million. Extrapolating to the national population (21.87 million) gave a prevalence of legal blindness of approximately 32892 or $0.15 \%$.

Conclusion Capture-recapture techniques can be used to determine the prevalence of blindness in whole populations. The calculated prevalence of blindness suggested that up to $30 \%$ of legally blind people may not be receiving available financial support and up to $60 \%$ were not accessing rehabilitation services.

The prevalence of blindness is a fundamental measure required effectively to target intervention programmes and to provide hard data against which progress can be evaluated. Most information on the prevalence of blindness is derived from blind registers or sampling surveys. ${ }^{12}$ The conventional approach to creating blind registers is by voluntary referrals from general practitioners and ophthalmologists. Reports have shown that underreporting of eligible patients was a significant problem particularly when there was no evaluation of the degree of ascertainment. ${ }^{34}$ Alternative methods for determining the prevalence of blindness have required sampling surveys of communities or complete population census data collection. ${ }^{56}$

Capture-recapture techniques were initially developed to estimate animal populations but have more recently been used and validated by epidemiologists to estimate the prevalence of medical conditions such as postoperative joint infections, brain injury, diabetes and pertussis in infants. ${ }^{7-11}$ The method utilises overlapping incomplete lists of affected individuals collated during repeated 'capture' exercises to estimate the size of the unsampled portion of the population. It also assess the degree of undercounting.

Our aim was to use capture-recapture methodology to determine the true prevalence of blindness in Western Australia. The results will enable us to improve the referral process by which people who are vision impaired are directed to support service providers, to monitor interventions and to predict future healthcare needs and costs.

\section{METHODS}

Legal blindness in Australia is defined as having a best corrected visual acuity of LogMAR greater than 1 or a visual field restriction of less than $10^{\circ}$ from central fixation or a combination of both reduced visual acuity and field loss resulting in the equivalent level of disability in the better eye.

In this study three separate 'capture' lists of people who were legally blind and resident in Western Australia (lists A, B and C), were collated over an 18-month period between April 2008 and September 2009. Western Australia has four tertiary-level hospitals including a paediatric hospital, each with public ophthalmology clinics, which contributed cases to the study. Both urban and rural-based consultant ophthalmologists, general practitioners and an optometrist enrolled patients in the study. Optometrists or ophthalmic nurses determined the level of vision loss. The diagnostic cause of vision loss and legal blindness status were determined by consultant ophthalmologists using full clinical notes, in all cases.

This study was approved by the human research ethics committees of Curtin University and participating hospitals.

List A was derived from the blind register held by the Association for the Blind of Western Australia. This recorded all individuals who were vision impaired or legally blind who were either selfreferred, or referred by ophthalmologists, optometrists or healthcare providers from anywhere within the state. It is a voluntary system of referral and has high diagnostic accuracy with a positive predictive value of 0.88 for legal blindness status. ${ }^{12}$ 
List B was collated over the 6-month period April to September 2008. It consisted of patients who were legally blind attending routine appointments at either public hospital outpatient eye clinics or selected consultant ophthalmologists' clinics or who were assessed by an optometrist.

Patients were not enrolled within a 7-day postoperative period. There were no age limits on being listed as legally blind. Listed patients gave consent for their identifiers (first name, middle name, last name, gender and date of birth) to be utilised for the specific purposes of this study. Patients were enlisted using standardised forms to record the level and cause of vision loss.

The third data list (C) was collected over a 6-week period August-September 2009. Eligible patients comprised those who were legally blind and attending either a tertiary hospital outpatient eye clinic or a consultant ophthalmologist's rooms or selected general medical practitioners' clinics. The methodology, selection and inclusion criteria were identical for this list as those for list B and were confirmed by ophthalmologists in all cases.

Deterministic unique matching with clerical review between lists (A, B and C) was achieved using available identifier fields: date of birth, gender, first and last names. All legally blind individuals listed on the blind register (list A) at 30 September 2009, with residential post codes within Western Australian state boundaries (post codes 6000-6999) were selected. Individuals with a matched identity on the state register of deaths, with a date of death before the end of the collection period were excluded. This facility was made possible by the Western Australian data linkage unit and permitted the accurate calculation of outmigration due to deaths in the blind register population.

Following the identification of the total number of individuals contained in the three lists and the matches identified between lists, the data were classified into seven cells of a three-way contingency table. The table has eight cells $\left(2^{3}\right)$ with the eighth cell corresponding to blind individuals who were absent from all three lists. The data are illustrated in a Venn diagram (figure 1) and in an incomplete contingency table (table 1). Incomplete contingency tables were used to estimate the number of individuals in the missing cell of the table using log-linear modelling. ${ }^{13} 14$

\section{Log-linear modelling}

The logarithm of the count in each cell of the table was modelled as a linear function of terms indicating the presence or

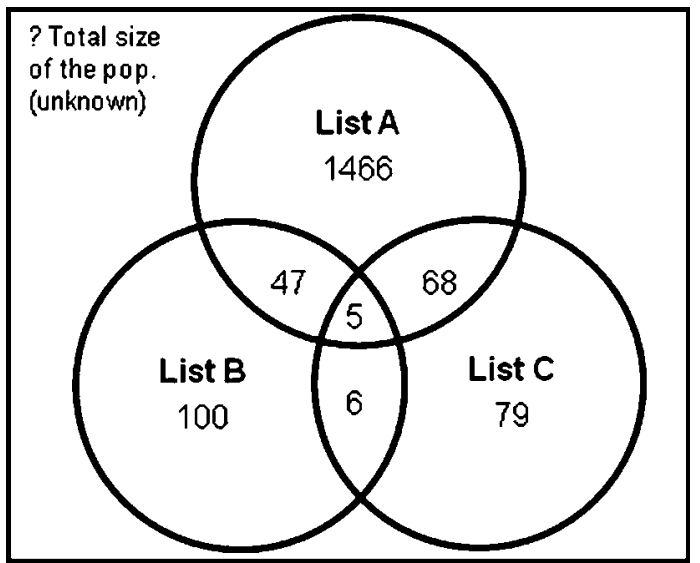

Figure 1 Venn diagram showing the distribution of 1771 individuals who were legally blind, to illustrate the overlap between the number of people who were blind and identified on each of the three lists: $A$, blind register; $B$, individuals attending routine hospital outpatient eye clinics 2008; and C, individuals attending ophthalmologist or general practitioner appointments 2009.
Table 1 Contingency table showing the distribution of 1771 individuals who were legally blind, between three lists

\begin{tabular}{llllll}
\hline & \multicolumn{2}{l}{ Recapture (C) present } & & \multicolumn{2}{l}{ Recapture (C) absent } \\
\cline { 2 - 3 } & $\begin{array}{l}\text { Capture (B) } \\
\text { present }\end{array}$ & $\begin{array}{l}\text { Capture (B) } \\
\text { absent }\end{array}$ & & $\begin{array}{l}\text { Capture (B) } \\
\text { present }\end{array}$ & $\begin{array}{l}\text { Capture (B) } \\
\text { absent }\end{array}$ \\
\hline Blind register (A) present & 5 & 68 & 47 & 1466 \\
Blind register (A) absent & 6 & 79 & 100 & -
\end{tabular}

$A$, blind register; $B$, individuals attending routine hospital outpatient eye clinics 2008; and $C$, individuals attending ophthalmologist or general practitioner clinical appointments 2009.

absence on each dimension (list). Pair-wise dependence of lists can be modelled as interaction terms between the relevant lists.

Explicitly: the log-linear model may be expressed using the following notation (following Chao et al): ${ }^{14}$

$$
\log \left(M_{a, b, c)}=u+u_{(a)}+u_{(b)}+u_{(c)}+u_{(a, b)}+u_{(a, c)}+u_{(b, c)}\right.
$$

Where $M_{a, b, c}$ represents the expected number of people identified in the combination of lists given by the indices $a, b$ and $c$; as the 'intercept' term in the linear model, $u$ represents the logarithm of the number not captured; other terms in the model represent the influence of relevant list membership (or 'joint' membership for interaction terms) on the expected number in that list (or combination of lists). It must be assumed that no three-way interaction term is present, so that the final (unknown) cell can be determined from the estimates of the main effects and twoway interaction terms in the model. Seven models were fitted to the data: one with no interactions (indicating that memberships of lists were independent of each other), three models with one two-way interaction term, and three models with two two-way interaction terms. Models fitted are shown in supplementary table 2, available online only.

The simplest model (least number of estimated parameters) in which the deviance indicated an adequate fit $\left(p>0.05, \chi^{2}\right.$ statistic with the appropriate degrees of freedom) was selected as the best model. The purpose of fitting the model was to estimate the number of people who were not captured in any of the lists. This number was estimated for each model, and was added to the number of people who were present in at least one list, to obtain an estimate of the total population of blind people. The $95 \%$ CI for the number not captured provide an indication of the reliability of the final population prevalence estimate.

Dependencies between source lists appear as statistically significant interaction terms in the log-linear model. The interaction term in the model attempts to correct for any dependence, and results in a model that fits the observed data better. The influence of this dependence on the estimate of people not listed is difficult to predict. However, more confidence can be placed in estimates that are derived from models that describe the data well. The interaction terms were included when they provide a significant improvement to the goodness of fit of the model. The model coefficients were estimated using the 'Genmod' procedure, SAS software program, version 9.1. Other analyses were carried out using PASW Statistics v18, (Microsoft).

Previous studies of the prevalence of blindness in Australia have been age restricted (50 years or more) in which blindness is more frequent. ${ }^{515}$ To compare the estimate of this study with previous estimates we recalculated the capture-recapture prevalence estimates, using the same matrices and models, after selecting only those people who were blind and aged 50 years or more, in each of the three collated lists.

All Australian adults who are legally blind are eligible for financial support in the form of a government pension. For the 
purposes of validation and comparison, we compared the estimated prevalence of blindness with the total number of blind pension recipients in the state, during the periods of data collection for this study. These de-identified data were made available by the Australian federal government offices of the Department of Families and Housing, Community Services and Indigenous Affairs.

\section{RESULTS}

This study identified 1771 individuals resident in Western Australia who were legally blind. There were 721 male and 1050 female individuals ranging in age from 1 year to 106 years at the end of the data collection period in 2009.

The blind register (list A) contained 1586 individuals who were confirmed as being alive and resident in the state of Western Australia. Eleven people on the blind register had recorded dates of death that could not be confirmed by the state register of deaths, and all were included in the analysis. Eight people from list $\mathrm{B}$ and one from list $\mathrm{C}$ died before the end of the study period (October 2009), and were not included in the final analysis. After clerical checking, 46 people on the blind register were excluded as they had no known residential post code in Western Australia (6000-6999) and were lost to follow-up.

The mean incidence of people who were legally blind added to the blind register over the previous 5 years (2004-8) was 320 per year or a cumulative incidence of 16 per 100000 population per year (figure 2A). The combined incidence of legally blind over the previous 24 years is shown in figure $2 \mathrm{~B} .{ }^{16}$
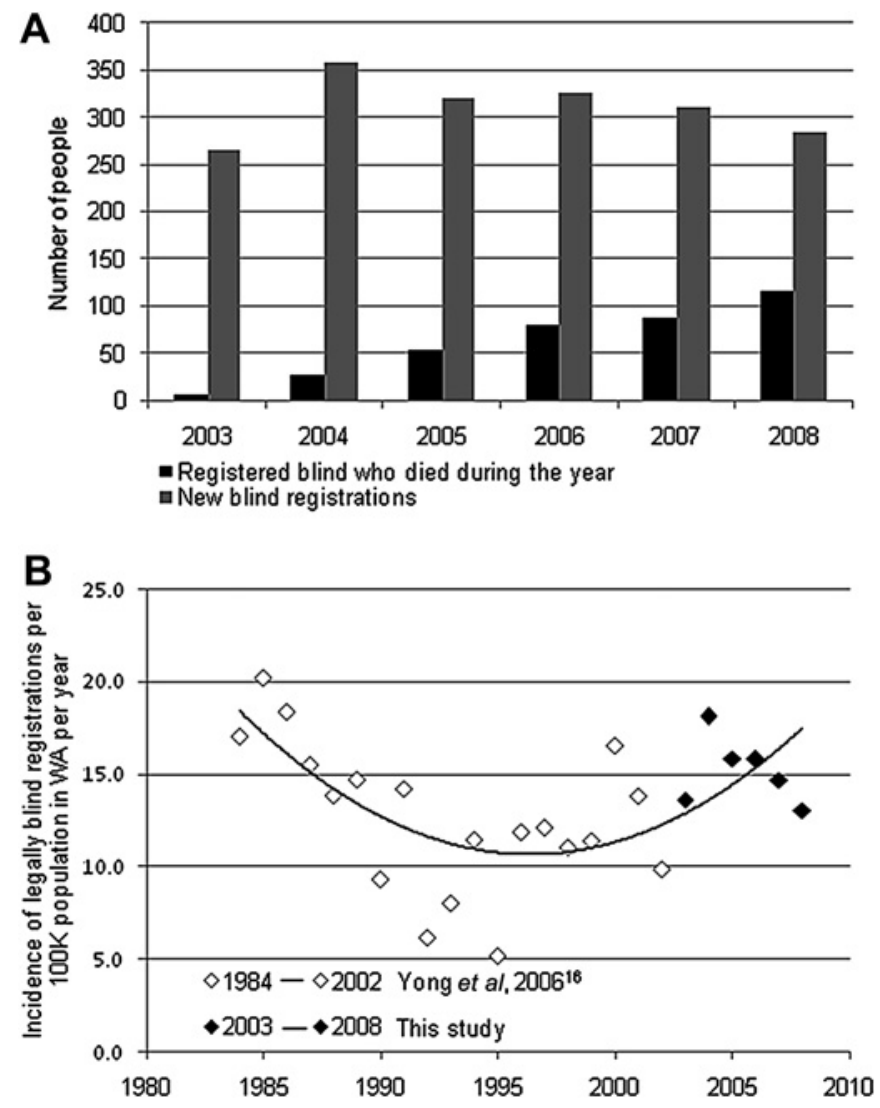

Figure 2 (A) Incidence in Western Australia (WA) of individuals who were registered as legally blind each year and the number of deaths in this population 2003-8. (B) Incidence of individuals who were legally blind registered annually in Western Australia per 100000 population over 24 years. Data from a previous study, Yong et $\mathrm{al}^{16}$ and from this study.
The distribution of people across the three 'capture' lists is shown in figure 1 . List B had 158 people who were blind, of which 100 were not present on either of the other lists (A and C). Thirty-seven were nursing home residents and of these, five were matched to people on list A. List C contained 158 people who were blind, of whom 79 were not present on either list A or list B. No nursing home residents were identified on list C. Five people were present on all three lists and six were present on both list B and list C.

Using log-linear modelling the simplest model, with no interactions between the three lists, led to a poor fit with the data $(p=0.021)$. The model with one interaction term (3) showed the best fit (deviance 2.63, $p=0.268$ ). The calculated estimate of the 'uncaptured' portion of the blind population was 1613. Together with the previous 1771 'captured', this provided a best estimate of the total state population of legally blind in 2009 as 3384 (95\% CI 2947 to 3983) (see supplementary table 3, available online only).

An age restricted (50 years or more) population prevalence estimate was also calculated in the same way (see supplementary figure 3, available online only). Using the same model (3) with a single interaction and the best fit $(\mathrm{p}=0.142)$, we obtained an estimate of the number of people who were blind to be 2859 ( $95 \%$ CI 2470 to 3408 ) or $0.43 \%$ of the state population aged 50 years or more $(n=666009)^{17}$ (see supplementary table 4 , available online only).

Extrapolating these results to a national level, the prevalence of legal blindness in Australia in 2009 was 32892 (95\% CI 28645 to 38715 ) equivalent to $0.15 \%$ of the national population of 21.9 million. The age restricted ( $\geq 50$ years) prevalence of blindness was $0.4 \%$ (95\% CI $0.37 \%$ to $0.51 \%$ ).

The estimated prevalence of people who were blind in Western Australia exceeded both the total number of recipients of the blind pension $(n=2244)$ and the number registered for support with the Association for the Blind of Western Australia (see supplementary figure 4, available online only).

\section{DISCUSSION}

This is the first study that we are aware of to use capture and recapture mathematics to calculate the prevalence of blindness in a whole population. We estimated that 3384 (95\% CI 2947 to 3983 ) or $0.15 \%$ of the Western Australia population of 2.25 million were legally blind at the end of September 2009. This is higher than either the number receiving the government blind pension or those registered for support from the Association for the Blind of Western Australia.

Comparing this prevalence estimate with previous crosssectional Australian studies, we found that the results were remarkably similar. Both the Melbourne Visual Impairment Project $^{18}$ and the Blue Mountains Eye Study ${ }^{5}$ reported a prevalence of legal blindness of $0.5 \%$ in the population aged 50 years or more. This suggests that the capture-recapture technique is a valid and highly cost-effective method compared with traditional cross-sectional, population-based surveys for determining disease prevalence.

The strengths of this study are the moderately stable and relatively isolated population. It was recently shown that the Western Australian population is representative of the Australian population as a whole. ${ }^{19}$ Therefore, data from this study can reasonably be extrapolated to the national population of Australia. All individuals who were legally blind were included in this estimate, with no age restrictions. A prevalence estimate has been obtained from a disparate and low prevalence population 
that is comparable to previous published estimates derived from large population sampling surveys.

The study was limited by two of the four assumptions of capture-recapture estimations: equal capture probabilities and a closed population (no births, deaths or migration). It is commonly accepted that this later assumption can rarely be fulfilled in human applications. ${ }^{20}$ The provision of medical services in rural Western Australia (geographical area 2.65 million $\mathrm{km}^{2}$ ) is limited, and as a result data collection from the very remote regions of the state was low, but not zero. In addition, people living in residential nursing care facilities, although not specifically excluded, were less likely to attend hospital or other clinical appointments and therefore the probability of being 'captured' was not equal when compared with others in the community. As remotely isolated people and nursing home residents were previously found to have a higher prevalence of blindness than the general population ${ }^{621-23}$ this would result in an underestimation of the true blind population.

These results have shown that relevant government and community agencies are unaware of many people who are legally blind. While it is possible that people who are blind may choose not to claim income support, may be financially independent or may not wish to be identified as legally blind, these results suggest that there may be up to $35 \%$ more people who are legally blind and eligible for financial support than are currently in receipt of a blind pension in Western Australia. We also found that only one third of individuals who are legally blind have ever been referred to or have visited the only provider of support services in the state, leaving $60 \%$ of individuals without rehabilitation support. Understanding the full extent of the burden of blindness will help to improve the targeting of appropriate rehabilitation programmes with special emphasis on psychological issues, increasing mobility and social connectivity.

This capture-recapture study has shown that it is a relatively efficient and cost-effective method for the accurate estimation of the prevalence of legal blindness.

Acknowledgements The authors would like to thank all the people who were blind who agreed to support this study. They also acknowledge the clinical and medical staff who assisted with the data collection.

Funding The Eye Surgery Foundation, Perth, Western Australia and Pfizer Australia provided unrestricted research grants with no role in the design or conduct of this research.

\section{Competing interests None.}

Ethics approval This study was approved by the human research ethics committees of Curtin University and participating hospitals.

Contributors The following are all members of the Eye and Vision Epidemiology Research (EVER) group and this project was part of the Epidemiology of Blinding Eye Disease Study (EBEDS). Author contributions to this paper: JC: Overall study co-ordination. Responsible for patient contact and clinic surveys. Responsible for data entry, data base security and validation checking. Carried out data analysis. Drafted, formatted and critically revised manuscript for publication. WHM: Clinical consultant ophthalmologist. Substantial contribution to conception of study design and clinical support for the project. Carried out clinical assessments of patients. Critically reviewed the manuscript providing important intellectual content. NM: Clinical consultant ophthalmologist. Substantial contribution to conception of study design and clinical support for the project. Carried out clinical assessments of patients. Critically reviewed the manuscript providing important intellectual content. GL: Clinical consultant paediatric ophthalmologist. Substantial clinical support for the project. Critically reviewed the manuscript providing important intellectual content. RP: Biostatistician, providing advice and analysis on the capture-recapture methodology. AM: Computer programmer with responsibility for data extraction and data management. AC: Clinician and ophthalmic registrar who assisted with patient assessments and statistical advice. JN: Ophthalmic registrar who assisted with patient assessments. MC: Chief executive officer of the Association for the Blind of Western Australia (ABWA). Data custodian of ABWA client information. JS: Chief investigator with overall responsibility for project management. Critically reviewed the manuscript.

Provenance and peer review Not commissioned; externally peer reviewed.

\section{REFERENCES}

1. Farber MD. National Registry for the Blind in Israel: estimation of prevalence and incidence rates and causes of blindness. Ophthalmic Epidemiol 2003;10:267-77.

2. Finger RP, Fimmers R, Holz FG, et al. Prevalence and causes of registered blindness in the largest federal state of Germany. $\mathrm{Br} J$ Ophthalmol 2011;95:1061-7.

3. Kahn HA, Moorhead HB. Statistics on Blindness in the Model Reporting Area: 1969-1970. Washington, DC: Department of Health, Education and Welfare, US Public Health Service and National Institutes of Health, 1973.

4. Robinson R, Deutsch J, Jones HS, et al. Unrecognised and unregistered visual impairment. Br J Ophthalmol 1994;78:736-40.

5. Attebo K, Mitchell P, Smith W. Visual acuity and the causes of visual loss in Australia: the Blue Mountains Eye Study. Ophthalmology 1996;103:357-64.

6. Taylor H, Xie J, Fox S, et al. The prevalence and causes of vision loss in indigenous Australians: the National Indigenous Eye Health Survey. Med J Aust 2010:192:312-18.

7. Hook EB, Regal RR. Capture-recapture methods in epidemiology: methods and limitations. Epidemiol Rev 1995;17:243-64.

8. Huotari K, Lyytikäinen 0, Ollgren J, et al. Disease burden of prosthetic joint infections after hip and knee joint replacement in Finland during 1999-2004: capture-recapture estimation. J Hosp Infect 2010;75:205-8.

9. Schootman M, Harlan M, Fuortes L. Use of capture-recapture method to estimate severe traumatic brain injury rates. J Trauma 2000;48:70-5.

10. Morris AD, Boyle DI, MacAlpine R, et al. The diabetes audit and research in Tayside Scotland (darts) study: electronic record linkage to create a diabetes register. BMJ 1997;315:524-8.

11. Somerville RL, Grant CC, Grimwood K, et al. Infants hospitalised with pertussis: estimating the true disease burden. J Paediatr Child Health 2007;43:617-22.

12. Crewe JM, Morgan WH, Morlet N, et al. Assessing the diagnostic validity of a blind register. Clin Exp Ophthalmol 2011;39:494-500.

13. International Working Group for Disease Monitoring and Forecasting. Capture-recapture and multiple-record systems estimation I: history and theoretica development. Am J Epidemiol 1995:142:1047-58.

14. Chao A, Tsay PK, Lin S-H, et al. The applications of capture-recapture models to epidemiological data. Stat Med 2001;20:3123-57.

15. Taylor H, Keeffe J, Vu H, et al. Vision loss in Australia. Med J Aust 2005; 182:565-8

16. Yong VK, Morgan WH, Cooper RL, et al. Trends in registered blindness and its causes over 19 years in Western Australia. Ophthalmic Epidemiol 2006;13:35-42.

17. Australian Bureau of Statistics. 2010. http://www.abs.gov.au/ausstats/abs@. nsf/mf/3101.0 Australian demographic statistics (accessed Sep 2009).

18. VanNewkirk MR, Weih L, McCarty CA, et al. Cause-specific prevalence of bilateral visual impairment in Victoria, Australia: the visual impairment project. Ophthalmology 2001;:108:960-7.

19. Clark A, Preen DB, Ng JQ, et al. Is Western Australia representative of other Australian states and territories in terms of key socio-demographic and health economic indicators? Aust Health Rev 2010;34:210-15.

20. Hook EB, Regal RR. Accuracy of alternative approaches to capture-recapture estimates of disease frequency: internal validity analysis of data from five sources. Am J Epidemiol 2000;152:771-9.

21. Owsley C, McGwin G, Scilley K, et al. The visual status of older persons residing in nursing homes. Arch Ophthalmol 2007;125:925-30.

22. de Winter LJM, Hoyng CB, Froeling PG, et al. Prevalence of remediable disability due to low vision among institutionalised elderly people. Gerontology 2004; 50:96-101.

23. Evans JR, Fletcher AE, Wormald RP, et al. Prevalence of visual impairment in people aged 75 years and older in Britain: results from the MRC trial of assessment and management of older people in the community. Br J Ophthalmol 2002;86:795-800. 


\section{BjO \\ Prevalence of blindness in Western Australia: a population study using capture and recapture techniques}

Julie Crewe, William H Morgan, Nigel Morlet, et al.

$\mathrm{Br} J$ Ophthalmo/ published online November 17, 2011

doi: 10.1136/bjophthalmol-2011-300908

Updated information and services can be found at:

http://bjo.bmj.com/content/early/2011/11/16/bjophthalmol-2011-300908.full.html

These include:

Data Supplement "Supplementary Data"

http://bjo.bmj.com/content/suppl/2011/11/17/bjophthalmol-2011-300908.DC1.html

References This article cites 21 articles, 8 of which can be accessed free at: http://bjo.bmj.com/content/early/2011/11/16/bjophthalmol-2011-300908.full.html\#ref-list-1

$\mathbf{P}<\mathbf{P} \quad$ Published online November 17, 2011 in advance of the print journal.

Email alerting

Receive free email alerts when new articles cite this article. Sign up in service the box at the top right corner of the online article.

Notes

Advance online articles have been peer reviewed, accepted for publication, edited and typeset, but have not not yet appeared in the paper journal. Advance online articles are citable and establish publication priority; they are indexed by PubMed from initial publication. Citations to Advance online articles must include the digital object identifier (DOIs) and date of initial publication.

To request permissions go to:

http://group.bmj.com/group/rights-licensing/permissions

To order reprints go to:

http://journals.bmj.com/cgi/reprintform

To subscribe to BMJ go to:

http://group.bmj.com/subscribe/ 